International Mathematical Forum, Vol. 8, 2013, no. 26, 1295 - 1305

HIKARI Ltd, www.m-hikari.com

http://dx.doi.org/10.12988/imf.2013.3593

\title{
Subperiodic Rings with Conditions on Extended Commutators
}

\author{
Adil Yaqub \\ Department of Mathematics \\ University of California \\ Santa Barbara, CA 93106, USA \\ yaqub@math.ucsb.edu
}

Copyright (C) 2013 Adil Yaqub. This is an open access article distributed under the Creative Commons Attribution License, which permits unrestricted use, distribution, and reproduction in any medium, provided the original work is properly cited.

\begin{abstract}
Let $R$ be a ring with Jacobson radical $J$ and with center $C$. Let $P$ be the set of potent elements $x$ for which $x^{k}=x$ for some integer $k>1$. Let $N$ be the set of nilpotents. A ring $R$ is called subperiodic if $R \backslash(J \cup C) \subseteq N+P$. We consider the commutativity behavior of a subperiodic ring with some constraint involving extended commutators.
\end{abstract}

\section{Mathematics Subject Classification: 16U80, 16D70}

Keywords: subperiodic ring, Jacobson radical, primitive ring, extended commutator, potent element, subdirectly irreducible ring, subdirect sum, commutator ideal

Throughout $R$ is a ring with nilpotents $N$, and with Jacobson radical $J$ and center $C$. The extended commutator $[x, y]_{k}$ is defined inductively by $[x, y]_{1}=$ $[x, y]=x y-y x$ and $[x, y]_{k}=\left[[x, y]_{k-1}, y\right]$ for all postive integers $k>1$. An element $x$ is called potent if $x^{k}=x$ for some integer $k>1$. The ring $R$ is called subperiodic if $R \backslash(J \cup C) \subseteq N+P$, where $P$ is the set of potent elements. 
In preparation for the proofs of the main theorems, we state the following lemmas.

Lemma 1 ([1]). Suppose $R$ is a ring in which each element is central or potent. Then $R$ is commutative.

Lemma 2 ([2]). Let $R$ be a ring such that both $J$ and $R / J$ are commutative. Then $N$ is an ideal and $R / N$ is commutative.

Lemma 3 ([3]). Suppose that $R$ is a ring such that for every element $x$ in $R$, there exists an integer $n>1$ such that $x-x^{n}$ is in the center of $R$. Then $R$ is commutative.

Lemma 4. Suppose $R$ is any ring such that $[x, y]$ commutes with $x$. Then, for all positive integers $n,\left[x^{n}, y\right]=n x^{n-1}[x, y]$.

This lemma is readily proved by induction.

Lemma 5. Suppose $R$ is a subperiodic ring, and suppose that $\sigma: R \rightarrow R_{i}$ is a homomorphism of $R$ onto a ring $R_{i}$. Suppose, further, that the set $N$ of nilpotents of $R$ is an ideal. Then, the set $N_{i}$ of nilpotents of $R_{i}$ is contained in $\sigma(J) \cup C_{i}$, where $C_{i}$ is the center of $R_{i}$.

Proof. By contradiction. Suppose $d_{i} \in N_{i}, d_{i} \notin \sigma(J), d_{i} \notin C_{i}$. Let $\sigma(d)=d_{i}$, $(d \in R)$. Then, $d \notin(J \cup C)$, and hence $d=a+b, a \in N$, b potent with $b^{k}=b$ for some integer $k>1$. Hence,

$$
d-a=b=b^{k}=(d-a)^{k} .
$$

Since $N$ is an ideal and $a \in N$, we conclude that $d-d^{k} \in N$, and hence

$$
\text { For all } d \in R \backslash(J \cup C), d-d^{k} \in N, k>1 \text {. }
$$

Let $d_{i}^{n}=0$ (snce $\left(d_{i} \in N_{i}\right)$. Since $d \notin(J \cup C)$, therefore by $(1), d-d^{k} \in N$, $k>1$. Since $N$ is an ideal,

$$
\left(d-d^{k}\right)+d^{k-1}\left(d-d^{k}\right)+\cdots+\left(d^{k-1}\right)^{n-1}\left(d-d^{k}\right) \in N,
$$

which implies (as is readily verified)

$$
d-d^{n+1} d^{n(k-2)} \in N
$$

Therefore, by (2),

$$
d_{i}-d_{i}^{n+1} d_{i}^{n(k-2)} \in \sigma(N) .
$$

Since $d_{i}^{n}=0$, (3) implies that $d_{i} \in \sigma(N) \subseteq \sigma(J)(N \subseteq J$ follows from the fact that $N$ is an ideal). So $d_{i} \in \sigma(J)$, contradiction. This proves the lemma. 
Lemma 6. Suppose $R$ is a subperiodic ring with central idempotents, and suppose $N$ is an ideal. Let $R_{i}$ be a subdirectly irreducible ring, and let $\sigma: R \rightarrow R_{i}$ be an onto homomorphism. Then $R_{i}$ is of one of the following two types:

Type 1: $R_{i}=\sigma(J) \cup C_{i}, C_{i}=$ center of $R_{i}$, or

Type 2: $R_{i}=\sigma(J) \cup C_{i} \cup U_{i}, U_{i}=$ the set of units of $R_{i}$. (Type 1 holds when $1 \notin R_{i}$, while type 2 holds when $1 \in R_{i}$.)

Proof. Write $R$ as a subdirect sum of subdirectly irreducible rings $R_{i}$. By (1), we see that for all $d \in R, d \in J$ or $d \in C$ or $d-d^{k} \in N, k>1$. Moreover, if $d-d^{k} \in N$, say $\left(d-d^{k}\right)^{q}=0$, then $d^{q}=d^{q+1} g(d)$ for some $g(x) \in \mathbb{Z}[x]$, and hence

$$
d^{q}=d^{q+1} g(d)=d^{q}(d g(d))=d^{q}(d g(d))^{2}=\ldots=d^{q} e,
$$

where $e=(d g(d))^{q}$ is an idempotent, and thus by (1), we see that

For all $d \in R, d \in J$ or $d \in C$ or $d^{q}=d^{q} e, e^{2}=e \in d \mathbb{Z}[d], e \in C$.

In view of the homomorphism $\sigma: R \rightarrow R_{i}$, let $x_{i} \in R_{i}$ and let $\sigma(x)=x_{i}$, $(x \in R)$. By hypothesis, any idempotent $e \in R$ is central in $R$, and hence $e_{i}=\sigma(e)$ is central in $R_{i}$. By (4), we conclude that

For all $d_{i} \in R_{i}, d_{i} \in \sigma(J)$ or $d_{i} \in C_{i}$ or $d_{i}^{q}=d_{i}^{q} e_{i}, e_{i}^{2}=e_{i} \in d_{i} \mathbb{Z}\left[d_{i}\right], e_{i} \in C_{i}$

Since $e_{i}$ is a central idempotent in the subdirectly irreducible ring $R_{i}, e_{i}=0$ or $e_{i}=1$. If $R_{i}$ does not have an identity, then by (5) and Lemma $5, R_{i}$ is as described in type 1 (since $e_{i}=0$ ). On the other hand, if $1 \in R_{i}$, then $R_{i}$ is as described in type 2 , since $e_{i} \in d_{i} \mathbb{Z}\left[d_{i}\right]$. This proves the lemma, since in the latter case, $e_{i}=\left(d_{i} g\left(d_{i}\right)\right)^{q}=1$ implies that $d_{i}$ is indeed a unit in $R_{i}$.

We are now in a position to prove the main theorems.

Theorem 1. Suppose $R$ is a subperiodic ring with Jacobson radical J, and suppose that $J$ is commutative. Suppose, further, that for all $x, y$ in $R$, there exist positive integers $k, l, m$ depending on $x, y$ such that

$$
x^{k} y^{l}[x, y]_{m} \text { is in the center } C,(x, y \text { in } R) \text {. }
$$

Then $N$ is an ideal and $R / N$ is commutative, and thus $R$ has a nil commutator ideal.

Proof. Let $S=R / J$. Then, by definition,

every noncentral element of $S$ is a sum of a nilpotent and potent element. (7) 
Since $S=R / J, S$ is a subdirect sum of primitive rings $S_{i}$.

Case 1. $S_{i}$ is a division ring. Since (7) holds in $S_{i}$, every element of $S_{i}$ is either central or potent, and hence by Lemma $1, S_{i}$ is commutative.

Case 2. $S_{i}$ is a primitive ring which is not a division ring. Since condition (6) holds in $S_{i}$, and since this condition is satisfied by all subrings and by all homomorphic images of $S_{i}$, it follows, by Jacobson's Density Theorem [[4]; p.33], that for some division ring $D$ and some integer $q>1$, the complete matrix ring $D_{q}$ also satisfies (6). This, however, is false, as can be seen by taking $x=E_{11}+E_{12}, y=E_{11}$ in $D_{q}$. This implies that $S$ is a subdirect sum of commutative division rings, and hence

$$
R / J \text { is commutative. }
$$

Thus, both $J$ and $R / J$ are commutative, and hence by Lemma $2, N$ is an ideal and $R / N$ is commutative, which proves the theorem.

Theorem 2. Suppose $R$ is a subperiodic ring with identity and suppose that $J$ is commutative. Suppose, further, that for all $x, y$ in $R$, there exist positive integers $k, l, m$ depending on $x, y$ such that

$$
x^{k} y^{l}[x, y]_{m} \text { is in the center } C,(x, y \text { in } R) \text {. }
$$

Then $R$ is commutative.

Proof. First, we show that

All idempotents of $R$ are central.

To prove this, let $e^{2}=e, x \in R$, and let $f=e+e x-e x e$. Then $f^{2}=f$, ef $=$ $f, f e=e$. By setting $x=f, y=e$ in (9), we obtain $e x=e x e$ (as is readily verified). Repeating this argument with $e$ now replaced by $1-e$, we see that $x e=e x e$, and hence $e$ is central, proving (10). Moreover,

$$
N \text { is an ideal (by Theorem 1). }
$$

As is well known,

$$
R \cong \text { a subdirect sum of subdirectly irreducible rings } R_{i} \text {. }
$$

Let $\sigma: R \rightarrow R_{i}$ be the natural homomorphismm of $R$ onto $R_{i}$. In view of Lemma 6 and (10), (11), (12), we see that

$$
R_{i}=\sigma(J) \cup C_{i} \cup U_{i}\left(C_{i}=\text { center of } R_{i}, U_{i}=\text { set of units in } R_{i}\right)
$$


Our next goal is to prove that

The set $N_{i}$ of nilpotents of $R_{i}$ is an ideal.

To prove this, let $a_{i} \in N_{i}, x_{i} \in R$. Then, by (13), $a_{i} x_{i} \in \sigma(J) \cup C_{i} \cup U_{i}$. If $a_{i} x_{i} \in C_{i}$, then $a_{i} x_{i} \in N_{i}$. Also, if $a_{i} x_{i} \in \sigma(J)$, then $\left(a_{i} x_{i}\right)^{2} \in(\sigma(J))^{2} \subseteq C_{i}$ (since $\sigma(J)$ is a commutative ideal), which implies that, for some $y_{i} \in R_{i}$, $\left(\left(a_{i} x_{i}\right)^{2}\right)^{k}=\left(a_{i} x_{i}\right)^{2}\left(a_{i} x_{i}\right)^{2} \ldots\left(a_{i} x_{i}\right)^{2}=a_{i}^{k} y_{i}$ for all postive integers $k$. Since $a_{i} \in N_{i}$, let $a_{i}^{k}=0$ in the above equation to obtain $\left(a_{i} x_{i}\right)^{2 k}=a_{i}^{k} y_{i}=0$, which implies that $a_{i} x_{i} \in N_{i}$. Finally, suppose $a_{i} x_{i}=u_{i}$, where $u_{i}$ is a unit in $R_{i}$, and let $a_{i}^{\delta}=0, \delta$ minimal. If $\delta=1$, then $a_{i} x_{i}=0 \in N_{i}$. Suppose then that $\delta>1$. Note that

$$
0=a_{i}^{\delta} x_{i}=a_{i}^{\delta-1}\left(a_{i} x_{i}\right)=a_{i}^{\delta-1} u_{i},\left(u_{i} \text { is a unit }\right)
$$

and hence $a_{i}^{\delta-1}=0$, contradicting the minimality of $\delta$. This contradiction shows that $a_{i} x_{i} \in N_{i}$. Similarly $x_{i} a_{i} \in N_{i}$. Moreover, by Lemma 5 , $N_{i} \subseteq \sigma(J) \cup C_{i}$ (see (11)), and hence $N_{i}$ is commutative (since $J$ is commutative) and (14) is proved. We claim that

$$
\text { For all } a_{i} \in \sigma(J), b_{i} \in R_{i},\left[a_{i}, b_{i}\right]_{q}=0 \text { for some } q \geq 1 \text {. }
$$

To prove this, note that (15) is trivially satisfied if $b_{i} \in C_{i}$ or if $b_{i} \in \sigma(J)$, and hence by (13),

$$
b_{i} \text { is a unit in } R_{i} \text {. }
$$

Note that $R_{i}$ inherits (9) in $R$, and hence for some positive integers $k, l, m$, we have $\left(1+a_{i}\right)^{k} b_{i}^{l}\left[1+a_{i}, b_{i}\right]_{m} \in C_{i}$ (the center of $R_{i}$ ), which implies that

$$
\left(1+a_{i}\right)^{k} b_{i}^{l}\left[a_{i}, b_{i}\right]_{m} \text { commutes with } b_{i} .
$$

Moreover, $\left(1+a_{i}\right)^{k}=1+a_{i} g\left(a_{i}\right)$ for some $g(x) \in \mathbb{Z}[x]$, and hence by $(17)$,

$$
\left(1+a_{i} g\left(a_{i}\right)\right) b_{i}^{l}\left[a_{i}, b_{i}\right]_{m} \text { commutes with } b_{i} .
$$

Since $a_{i} \in \sigma(J),\left(a_{i} g\left(a_{i}\right)\right) b_{i}^{l}\left[a_{i}, b_{i}\right]_{m} \in(\sigma(J))^{2} \subseteq C_{i}$, and hence by (18),

$$
b_{i}^{l}\left[a_{i}, b_{i}\right]_{m} \text { commutes with } b_{i} .
$$

Thus,

$$
\left(b_{i}^{l}\left[a_{i}, b_{i}\right]_{m}\right) b_{i}=b_{i}\left(b_{i}^{l}\left[a_{i}, b_{i}\right]_{m}\right) .
$$


In view of (16), we see that $(20)$ implies that $\left[\left[a_{i}, b_{i}\right]_{m}, b_{i}\right]=0$, and thus, $\left[a_{i}, b_{i}\right]_{m+1}=0$, which proves $(15)$.

Recall that $\sigma: R \rightarrow R_{i}$ is the natural homomorphism of the ground $\operatorname{ring} R$ onto the subdirectly irreducible ring $R_{i}$. Our next objective is to prove that

$$
\sigma(J) \text { is contained in the center of } R_{i} \text {. }
$$

Suppose not. Then,

$$
\left[a_{i}, b_{i}\right] \neq 0 \text { for some } a_{i} \in \sigma(J), b_{i} \in R_{i}
$$

Since $\left[a_{i}, b_{i}\right] \neq 0$, not both $2 b_{i}$ and $3 b_{i}$ commute with $a_{i}$. Assume, without loss of generality, that

$$
\left[a_{i}, 2 b_{i}\right] \neq 0,\left(a_{i} \in \sigma(J), b_{i} \in R_{i}\right)
$$

In view of (13), we see that (22) and (23) imply that

$$
b_{i} \text { and } 2 b_{i} \text { are both units in } R_{i} \text {, since } \sigma(J) \text { is commutative. }
$$

Since $N$ is an ideal (see (11)), all the hypotheses of Lemma 5 hold in $R$, and hence by (1) of Lemma 5 ,

$$
\text { For all } d \in R, d \in J \text { or } d \in C \text { or } d-d^{k} \in N, k>1 \text {. }
$$

Note that, since $R_{i}$ inherits (25) from $R$, we have:

$$
\text { For all } d_{i} \in R_{i}, d_{i} \in \sigma(J) \text { or } d_{i} \in C_{i} \text { or } d_{i}-d_{i}^{k} \in N_{i}, k>1 \text {. }
$$

Moreover, since $\sigma(J)$ is commutative, (22) and (23) imply

$$
b_{i} \notin \sigma(J) \cup C_{i} \text { and } 2 b_{i} \notin \sigma(J) \cup C_{i} \text {. }
$$

By (26) and (27), we conclude that for some positive integers $k_{i}, l_{i}$

$$
b_{i}-b_{i}^{k_{i}} \in N_{i} \text { and }\left(2 b_{i}\right)-\left(2 b_{i}\right)^{l_{i}} \in N_{i}, k_{i}>1, l_{i}>1 \text {. }
$$

By (14), $N_{i}$ is an ideal of $R_{i}$. Let $\bar{x}_{i}=x_{i}+N_{i}$, for any $x_{i} \in R_{i}$. Then, by (28),

$$
\left(\bar{b}_{i}\right)^{k_{i}}=\bar{b}_{i} \text { and }\left(\overline{2}_{i}\right)^{l_{i}}=\overline{2}_{i}, k_{i}>1, l_{i}>1 \text {. }
$$


Note that by (29),

$$
\left(\bar{b}_{i}\right)^{\left(k_{i}-1\right)\left(l_{i}-1\right)+1}=\bar{b}_{i} \text { and }\left(\overline{2}_{i}\right)^{\left(k_{i}-1\right)\left(l_{i}-1\right)+1}=\overline{2}_{i} .
$$

Thus,

$$
2^{\left(k_{i}-1\right)\left(l_{i}-1\right)+1} \bar{b}_{i}=2^{\left(k_{i}-1\right)\left(l_{i}-1\right)+1}\left(\bar{b}_{i}\right)^{\left(k_{i}-1\right)\left(l_{i}-1\right)+1}=2 \bar{b}_{i} .
$$

Hence,

$$
\left(2^{\left(k_{i}-1\right)\left(l_{i}-1\right)+1}-2\right) \bar{b}_{i}=\overline{0} .
$$

Since $b_{i}$ is a unit (see (24)), $\bar{b}_{i}$ a unit also. Thus,

$$
\left(2^{\left(k_{i}-1\right)\left(l_{i}-1\right)+1}-2\right) \cdot \overline{1}=\overline{0}\left(k_{i}>1, l_{i}>1\right),
$$

which implies that

$$
\left(2^{\left(k_{i}-1\right)\left(l_{i}-1\right)+1}-2\right) \cdot 1 \text { is nilpotent, }
$$

and hence $R_{i}$ is not of zero characteristic. Since $R_{i}$ is subdirectly irreducible, we conclude that

$$
\text { Characteristic of } R_{i}=p^{k}, p \text { prime. }
$$

In view of (24), (29), and (30), it follows that the subring $<\bar{b}_{i}>$ generated by the unit $\bar{b}_{i}$ is a finite commutative ring with identity which has no nonzero nilpotents, and hence

$$
<\bar{b}_{i}>=\bigoplus_{j=1}^{t} G F\left(p^{k_{j}}\right), t \text { finite. }
$$

Let $\alpha=k_{1} k_{2} \ldots k_{t}$. Then, by (31) and (30), $\bar{b}_{i}^{p^{k \alpha}}=\bar{b}_{i}$, and thus by Lemma 5 and (11),

$$
b_{i}^{p^{k \alpha}}-b_{i} \in N_{i} \subseteq \sigma(J) \cup C_{i} .
$$

Returning to (15), let $\beta$ be the least positive integer such that

$$
\left[a_{i}, b_{i}\right]_{\beta}=0(\beta \text { minimal }), a_{i} \in \sigma(J) .
$$

We claim that

$$
\beta \leq 2
$$


Suppose not. Then $\beta>2$, and hence by (32),

$$
\left[b_{i}^{p^{k \alpha}}-b_{i},\left[a_{i}, b_{i}\right]_{\beta-2}\right]=0, a_{i} \in \sigma(J), b_{i} \in R_{i},
$$

which implies

$$
\left[\left[a_{i}, b_{i}\right]_{\beta-2}, b_{i}^{p^{k \alpha}}\right]=\left[\left[a_{i}, b_{i}\right]_{\beta-2}, b_{i}\right]
$$

By (33), we see that $\left[\left[a_{i}, b_{i}\right]_{\beta-2}, b_{i}\right]$ commutes with $b_{i}$, and hence by (36) and Lemma 4,

$$
p^{k \alpha} b_{i}^{p^{k \alpha}-1}\left[\left[a_{i}, b_{i}\right]_{\beta-2}, b_{i}\right]=\left[\left[a_{i}, b_{i}\right]_{\beta-2}, b_{i}\right],
$$

which implies that $\left.0=\left[\left[a_{i}, b_{i}\right]_{\beta-2}\right], b_{i}\right]$, by (30). Thus, $\left[a_{i}, b_{i}\right]_{\beta-1}=0$, which contradicts the minimality of $\beta$ (see (33)). This contradiction proves that $\beta \leq 2$, and thus

$$
\left[a_{i}, b_{i}\right] \text { commutes with } b_{i}
$$

By $(22), a_{i} \in \sigma(J)$, and hence by (32) and the fact that $\sigma(J)$ is commutative, we conclude that

$$
\left[a_{i}, b_{i}^{p^{k \alpha}}-b_{i}\right]=0
$$

which implies that

$$
\left[a_{i}, b_{i}^{p^{k \alpha}}\right]=\left[a_{i}, b_{i}\right]
$$

Combining (38), (37), (30), and Lemma 4, we obtain $\left[a_{i}, b_{i}\right]=0$, which contradicts (22). This contradiction proves (21).

To complete the proof, note that by (26), we have:

For all $d_{i} \in R_{i}, d_{i} \in \sigma(J)$ or $d_{i} \in C_{i}$ or $d_{i}-d_{i}^{k} \in N_{i}, k>1$.

Moreover, since $\sigma: R \rightarrow R_{i}$ is an onto homomorphism (see (12)) and since $N$ is an ideal (see (11)), it follows by Lemma 5 that $N_{i} \subseteq \sigma(J) \cup C_{i} \subseteq C_{i}$, by (21). Combining this with (21), (39), and Lemma 3 , we see that $R_{i}$ is commutative, and the ground ring $R$ is commutative. This proves the theorem.

The next theorem removes the hypothesis that $1 \in R$ in Theorem 2 .

Theorem 3. Suppose $R$ is a subperiodic ring and suppose $J$ is commutative. Suppose, further, that for all $x, y$ in $R$, there exist positive integers $k, l, m$ depending on $x$ and $y$ such that

$$
\text { Both } x^{k} y^{l}[x, y]_{m} \text { and }[x, y]_{m} x^{k} y^{l} \text { are central, }(x, y \text { in } R) \text {. }
$$

Then $R$ is commutative. 
Proof. First, we show that

All idempotents of $R$ are central.

As we saw at the beginning of the proof of Theorem 2,

$$
e x=e x e \text { for all idempotents } e \text { and all } x \text { in } R \text {. }
$$

(That proof did not assume that $R$ has an identity.) Next, we prove that

$$
x e=e x e \text { for all idempotents } e \text { and all } x \text { in } R \text {. }
$$

Let $f=e+x e-e x e, e^{2}=e$. Then $f^{2}=f$, ef $=e, f e=f$. By setting $x=e, y=f$ in the hypothesis " $[x, y]_{m} x^{k} y^{l}$ is central" in (40), we obtain (as is readily verified), $x e=e x e$, which proves (41). Also, by Theorem 1 ,

$N$ is an ideal.

Write $R$ is a subdirect sum of subdirectly irreducible rings $R_{i}$. Let $\sigma: R \rightarrow R_{i}$ be the natural homomorphism of $R$ onto $R_{i}$. In view of (41), (44), and Lemma $6, R_{i}$ must be of the following two types:

Type 1. $R_{i}=\sigma(J) \cup C_{i},\left(C_{i}=\right.$ center of $\left.R_{i}\right)$, or

Type 2. $R_{i}=\sigma(J) \cup C_{i} \cup U_{i},\left(U_{i}=\right.$ set of units in $\left.R_{i}\right)$.

Clearly, if $R_{i}$ is of type 1 , then $R_{i}$ is commutative, since $J$ is commutative. So we may assume that

$$
1 \in R_{i} \text { and } R_{i}=\sigma(J) \cup C_{i} \cup U_{i},\left(U_{i}=\text { set of units in } R_{i}\right) .
$$

We claim that

$$
\left.U_{i} \subseteq C_{i} \text { (center of } R_{i}\right)
$$

Suppose not. Let $u_{i} \in U_{i}, x_{i} \in R_{i}$ be such that $\left[u_{i}, x_{i}\right] \neq 0$. Let $d \in R$ be such that $\sigma(d)=u_{i}$. Since $\left[u_{i}, x_{i}\right] \neq 0, d \notin C$ (center of $R$ ). Also, if $d \in J$, then $u_{i}=\sigma(d) \in \sigma(J)$, and hence $\sigma(J)=R_{i}$ (since $u_{i}$ is a unit), which implies that $R_{i}$ is commutative (since $J$ is commutative), contradicting the fact that $\left[u_{i}, x_{i}\right] \neq 0$. This contradiction proves that $d \notin J$. The net result is: $d \notin(J \cup C)$, and hence

$$
d=a+b, a \in N, b \text { potent with } b^{k}=b, k>1 .
$$

Thus, $d-a=b=b^{k}=(d-a)^{k}, k>1$. Since $N$ is an ideal (see (44)) and $a \in N$ (see (47)), $d-d^{k} \in N$. Thus,

$$
d-d^{k} \in N, k>1, d \notin(J \cup C), d \in R .
$$


In view of (41), (44), (48), and (4) of Lemma 6, we conclude that, for some positive integer $q$,

$$
d^{q}=d^{q} e, e^{2}=e, e \text { central in } R(\text { by }(41))
$$

By above, $\sigma(d)=u_{i}$, and hence by $(49), u_{i}^{q}=u_{i}^{q} \sigma(e), u_{i}$ a unit, which implies that

$$
\sigma(e)=1,\left(e \in R, 1 \text { is the identity of } R_{i}\right) .
$$

Thus, by (49), (50), we see that

$$
e \text { is a central idempotent, } e \neq 0, e \in R \text {, }
$$

and hence $e R$ is a ring with identity $e$, which (as is readily verified) satisfies all the hypotheses of Theorem 2, and hence by Theorem 2,

$$
e R \text { is commutative. }
$$

Recall that $\sigma: R \rightarrow R_{i}$ is an onto homomorphism. Let $x_{i}, y_{i} \in R_{i}$ and let $\sigma(x)=x_{i}, \sigma(y)=y_{i},(x, y \in R)$. Then, by $(52),[e x, e y]=0$, and hence $[\sigma(e x), \sigma(e y)]=0$, which implies by $(50),[\sigma(x), \sigma(y)]=0$, and thus $\left[x_{i}, y_{i}\right]=0$. Therefore, $R_{i}$ is commutative, which contradicts the fact that $\left[u_{i}, x_{i}\right] \neq 0$ (see above). This contradiction proves (46). By (45) and (46), we conclude that $R_{i}=\sigma(J) \cup C_{i}$, and hence $R_{i}$ is commutative, (see type 1), which implies that the ground ring $R$ is commutative. This proves the theorem.

Remark 1. In (40), we could have replaced the integers $k, l, m$ in " $[x, y]_{m} x^{k} y$ " by the integers $k^{\prime}, l^{\prime}, m^{\prime}$, as a glance at the proof of (41) shows.

We conclude with the following:

Corollary 1. Suppose $R$ is a subperiodic ring and suppose $J$ is commutative. Suppose that for all $x, y$ in $R$, there exists a positive integer $m$, depending on $x$ and $y$, such that $[x, y]_{m}=0$. Then $R$ is commutative.

Related work appears in [5].

\section{References}

[1] H.E. Bell, A near-commutativity property for rings, Result. Math. 42 (2002), 28-31. 
[2] H.E. Bell, On Some commutativity theorems of Herstein, Arch. Math. 24(1973), 34-38.

[3] I.N. Herstein, A generalization of a theorem of Jacobson, III. Amer. J. Math. 75 (1953), 105-111.

[4] N. Jacobson, Structure of Rings, Amer. Math. Soc. Colloq. Publications, Vol. 37, Providence, RI 1964.

[5] A. Yaqub, Generalized J-rings, Periodicity, and Commutativity, Far East Journal of Math. Science. 59(2011), 113-126.

Received: May 2, 2013 